\title{
Possible key microRNAs and corresponding molecular mechanisms for atrial fibrillation
}

\author{
(D) Huili Zhang*,\#, (1) Guangming Yang**, \#, (D) Ning Zhong***, (D) Jun Shan****, \\ (10) Xiaona $\mathrm{Li}^{*}$, (1) Yanhai $\mathrm{Wu}^{* * *}$, (1) Yazhou $\mathrm{Xu}^{1}$, (1) Ye Yuan ${ }^{1}$ \\ Departments of *Pharmacy, and **Emergency, ***Cardiovasology, ****General Medicine, \\ The Second People's Hospital of Xinxiang; Henan-China \\ 'Henan Zhongping Genetic Technology Co., Ltd., Zhengzhou; Henan-China
}

\section{ABSTRACT}

Objective: We aimed to find crucial microRNAs (miRNAs) associated with the development of atrial fibrillation (AF), and then try to elucidate the possible molecular mechanisms of miRNAs in AF.

Methods: The miRNA microarray, GSE68475, which included 10 right atrial appendage samples from patients with persistent AF and 11 samples from patients with normal sinus rhythm, was used for the analysis. After data preprocessing, differentially expressed miRNAs were screened using limma. Target genes of miRNAs were predicted using miRWalk2.0. We then conducted functional enrichment analyses for miRNA and target genes. Protein-protein interaction (PPI) network and module analyses for target genes were performed. Finally, transcription factors (TFs)-target genes regulatory network was predicted and constructed.

Results: Seven genes, including CAMK2D, IGF2R, PPP2R2A, PAX6, POU3F2, YWHAE, and AP2A2, were targeted by TFs. Among these seven genes, CAMK2D (targeted by miR-31-5p), IGF2R (targeted by miR-204-5p), PAX6 (targeted by miR-223-3p), POU3F2 (targeted by miR-204-5p), YWHAE (targeted by miR-31-5p), and AP2A2 (targeted by miR-204-5p) belonged to the top 10 degree genes in the PPI network. Notably, MiR204-5p, miR-31-5p, and miR-223-3p had more target genes. Besides, CAMK2D was enriched in some pathways, such as adrenergic signaling in cardiomyocytes pathway and cAMP signaling pathway. YWHAE was enriched in the Hippo signaling pathway.

Conclusion: miR-31-5p played a crucial role in cardiomyocytes by targeting CAMK2D and $Y W H A E$ via cAMP and Hippo signaling pathways. miR204 was involved in the progression of AF by regulating its target genes IGF2R, POU3F2, and AP2A2. On the other hand, miR-223-3p functioned in AF by targeting PAX6, which was associated with the regulation of apoptosis in AF. This study would provide a theoretical basis and potential therapeutic targets for the treatment of AF. (Anatol J Cardiol 2020; 23: 324-33)

Keywords: atrial fibrillation, microRNAs, differentially expressed genes, pathways

\section{Introduction}

Atrial fibrillation (AF) is an abnormal heart rhythm characterized by irregular and rapid beating (1). The most common alterable risk factors for $\mathrm{AF}$ are valvular heart disease and high blood pressure (2). The prevalence rate of AF is approximately $1 \%$ in the general population, and goes up to $8 \%$ for individuals older than 80 years of age (3). The morbidity rate of AF is high, causing significant losses to society and individuals (4). However, the precise molecular mechanisms underlying $\mathrm{AF}$ have not been elucidated (5). Moreover, medical intervention for this disease is relatively limited (6). Warfarin is currently the most prescribed oral anticoagulant for the prevention of venous thromboembo- lism and systemic embolism in patients with AF. However, therapeutic management with warfarin is a challenge because of its narrow therapeutic index and the extensive interindividual and interethnic differences in dose requirements (7). Therefore, understanding the molecular mechanisms underlying $\mathrm{AF}$ may provide clues for the treatment of the disease.

MicroRNA (miRNA), a small non-coding RNA molecule, plays a role in RNA silencing and post-transcriptional gene expression regulation. Recently, some studies reported the significant role of miRNAs in regulating arrhythmogenesis and cardiac excitability in cardiac diseases, such as diabetic cardiomyopathy (8) and AF (9). Li et al. (10) identified several miR$\mathrm{NAs}$ and their target genes involved in the pathogenesis of AF

"These authors contributed equally to this work

Address for correspondence: Huili Zhang, MD, Department of Pharmacy, The Second People's Hospital of Xinxiang,

389 Hongli Avenue, Muye District, Xinxiang, 453002, Henan-China

Phone: +86-0373-3660566 E-mail: pyg7a8wii8aai@sina.com

Accepted Date: 27.02.2020 Available Online Date: 28.04.2020

(C) Copyright 2020 by Turkish Society of Cardiology - Available online at www.anatoljcardiol.com DOI:10.14744/AnatolJCardiol.2020.39483 
by analyzing microarrays data. Harling et al. (11) indicated that increased serum miR-483-5p levels might predict the risk of postoperative AF. The miR-29a-3p may play roles in the development of $\mathrm{AF}$ by downregulating L-type $\mathrm{Ca}^{2+}$ current (12). Furthermore, the significant roles of some miRNAs (e.g., miR328, miR-26, and miR-1) in the pathogenesis of AF have been validated $(9,13,14)$.

Morishima et al. (15) generated the dataset GSE68475 to profile miRNA expression in human atrial appendages, and they determined that miR-30d was essential for the electrical remodeling of AF. However, the possible molecular mechanisms of the miRNA in AF have not been elucidated. Therefore, we aimed to find crucial miRNAs associated with AF development, and then try to interpret the possible molecular mechanisms of miRNA in AF. This study purposed to provide a theoretical basis and potential therapeutic targets for the treatment of AF.

\section{Methods}

\section{Workflow of this study}

In the current study, the miRNA microarray GSE68475, which included 10 samples from patients with persistent AF and 11 samples from patients with normal sinus rhythm, was used for the analysis. After data preprocessing, differentially expressed miRNAs were screened. The target genes of miRNAs were predicted. We then conducted functional enrichment analyses for miRNA and target genes. Subsequently, protein-protein interaction (PPI) network and module analyses for target genes were performed. Finally, transcription factors (TFs)-target genes regulatory network was predicted and constructed.

\section{Microarray data}

The miRNA microarray GSE68475 (15) was downloaded from the gene expression omnibus (GEO, http://www.ncbi.nlm. nih.gov/geo// database. It included 10 right atrial appendage samples from patients with persistent AF (AF group, with a documented record of sustained AF for 6 months or longer) and 11 right atrial appendage samples from patients with normal sinus rhythm (NSR group, with no documented history of AF). Human right atrial appendage samples were collected from male patients aged $60-79$ years undergoing open-heart surgery at Oita University Hospital, who were included after excluding chronic heart failure, diabetes, inflammatory diseases, endocrine diseases, metabolic diseases, kidney diseases requiring hemodialysis, history of steroid treatment, and paroxysmal AF. Clinical data of patients, including blood pressure and history of medicine, are presented in Supplementary Table 1 [came from the original data of Morishima et al. (15), who generated the dataset GSE68475]. The platform was GPL15018 Agilent-031181 Unrestricted_Human_miRNA_V16.0_Microarray 030840 (Feature Number version).

\section{Data preprocessing}

For the original miRNA data, the limma (linear models for microarray data) (16) (Version 3.10.3, http://www.bioconductor. org/packages/2.9/bioc/html/limma.html) in R software (version 3.3.2) was used to perform background correction of expression values and normalized pre-processing of expression profile data, which included conversion of original data format, provision of missing value, background correction (MAS), and data standardization. Annotation was conducted using platform annotation files, and probes not matching to miRNAs were removed.

\section{Screening of differentially expressed miRNA}

Differentially expressed miRNAs between AF group and NSR group were screened using limma (16) (Version 3.10.3, http://www.bioc onductor.org/packages/2.9/bioc/html/limma. html). For each significantly differentially expressed miRNA, adj. $p$ value $<0.05$ and $\| \log$ fold change $(F C) \mid>0.585$. The heatmap was drawn using the $\mathrm{R}$ software pheatmap (17) Version 1.0.10, https://cran.r-project.org/web/packages/pheatmap/index.html).

\section{Prediction of miRNA-target genes}

We focused on analyzing the significantly differentially expressed top 10 upregulated and downregulated miRNAs. The miRNA-target genes were predicted using miRWalk2.0 (18) (http://zmf.umm.uni-heidelberg.de/apps/zmf/mirwalk2/). In the microRNA information retrieval system, the searching criteria were as follows: minimum seed length $=7, p$ value $<0.05$, and input parameters $=3^{\prime} U T R$. The searching databases were miRWalk (http://mirwalk.umm.uni-heidelberg.de/), miRanda (http://www.microrna.org/), miRDB (http://mirdb.org), miRMap (https://mirmap.ezlab.org/), Pictar2 (http://pictar.bio.nyu. edu), RNA22 (https://cm.jefferson.edu/rna22/Interactive/), and Targetscan (http://targetscan.org). The miRNA-target results were required to appear in seven databases at the same time. The miRNA-target genes regulatory network was constructed using Cytoscape (19) (version 3.2.0, http://www.cytoscape. $\operatorname{org} /$ ).

Functional enrichment analyses for miRNA and target genes

We performed the Kyoto Encyclopedia of Genes and $\mathrm{Ge}$ nomes (KEGG, https://www.kegg.jp/) (20) pathway enrichment analyses for miRNA using $\mathrm{R}$ software clusterProfiler (21). Furthermore, gene ontology (G0, http://geneontology.org/)-biological process (BP) (22) and KEGG (20) pathway enrichment analyses were conducted for genes targeted by miRNAs. Significant threshold values were $p$ value $<0.05$ and count value $\geq 2$.

\section{Protein-protein interaction network and module analyses for target genes}

The interactions between gene coding proteins were predicted using the STRING database (23) (Version 10.0, http://www. 


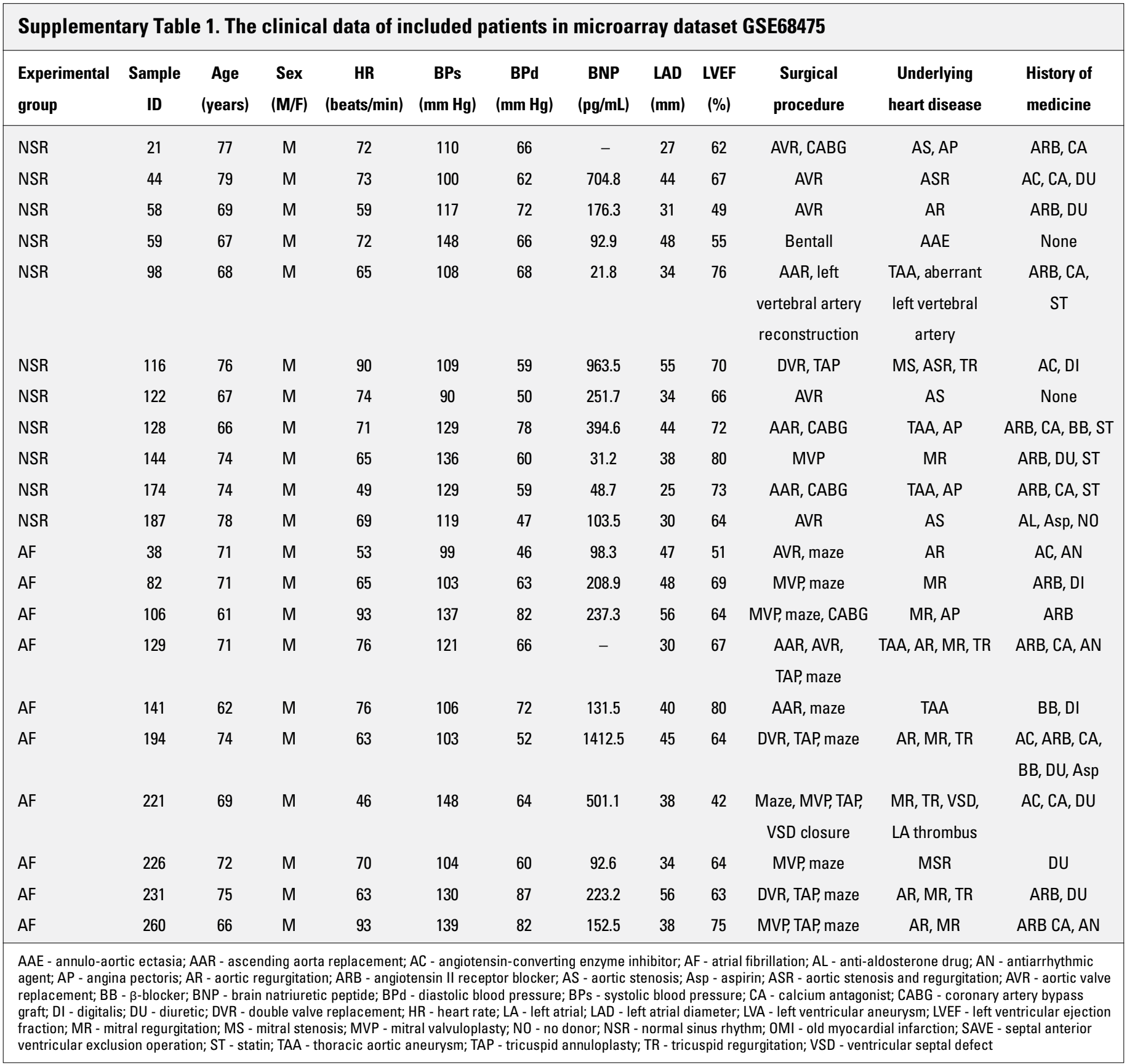

string-db.org/). The input gene sets were genes regulated by miRNAs, and the species were homo. PPI score was set as 0.4 (representing median confidence). The PPI network was constructed using Cytoscape (version 3.2.0).

Degree centrality was used to analyze the score of nodes in the network. The higher the node score, the more crucial the node was, and the more likely it was to be the key node.

The significantly enriched modules were analyzed using Сytoscape plug-in MCODE (24) (version 1.4.2, http://apps.cytoscape. org/apps/MCODE). The threshold value was score $\geq 5$.

GO-BP and KEGG pathway enrichment analyses were performed for key nodes of the top 10 degree and module. The threshold value was score $\geq 5$.
Prediction of transcription factors-target genes regulatory networks

Based on WebGestalt (25) (http://www.webgestalt.org/), TFtarget enrichment was predicted for the top 10 degree and module genes using overrepresentation enrichment analysis (ORA). The TFs-target genes regulatory network was constructed using Cytoscape.

\section{Results}

\section{Differential expression analyses}

After preprocessing for miRNA data, according to sample dispersion, the samples 01, 05 in the AF group and 03, 08 in the 
NSR group were removed, and $8 \mathrm{AF}$ samples and 9 NSR samples were obtained.

After screening, 49 (30 upregulated and 19 downregulated) significantly differentially expressed miRNAs were obtained. The heatmap for differentially expressed miRNAs is presented in Figure 1.

\section{miRNA-target genes regulatory network}

After the prediction through miRWalk2.0, overall 244 miRNA-target genes pairs, including 12 miRNAs (3 upregulated and

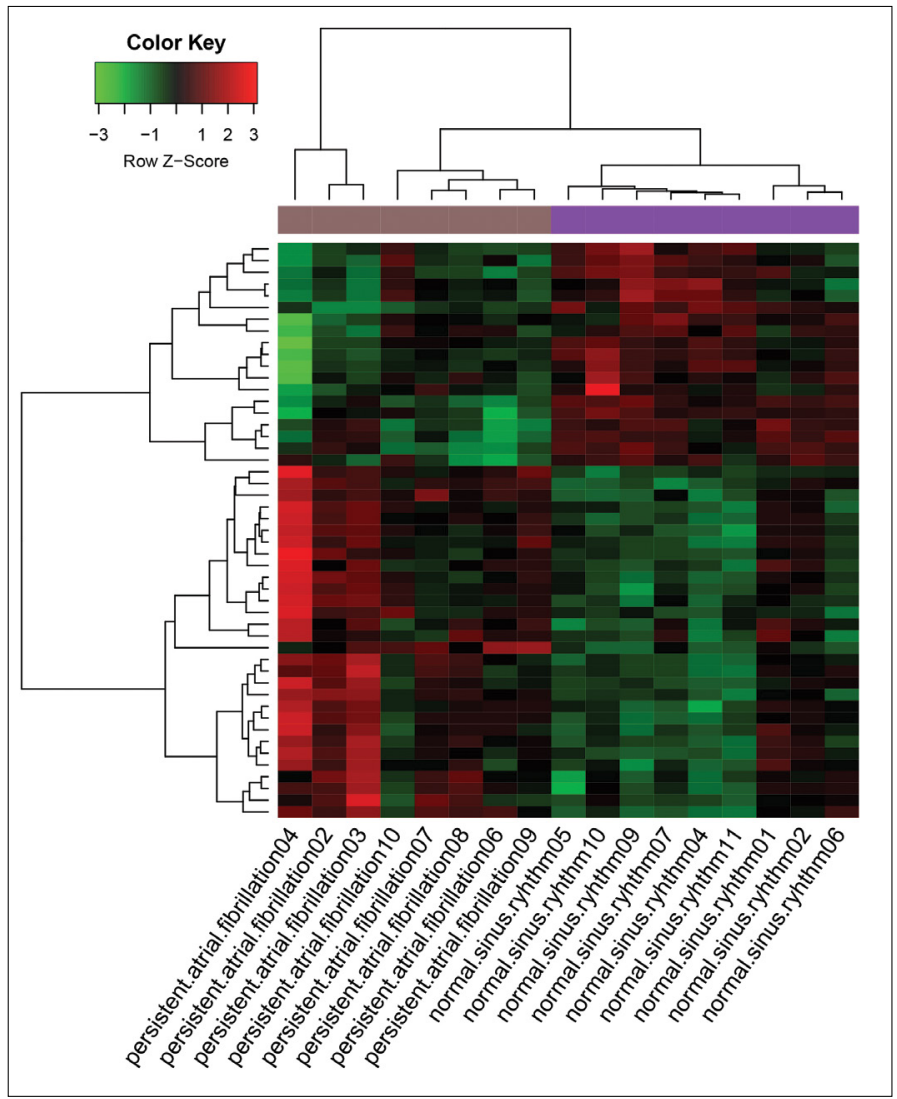

Figure 1. The heatmap for differentially expressed miRNAs
9 downregulated), were obtained. The miRNA-target genes regulatory network (244 nodes) is presented in Figure 2. For example, miR-204-5p targeted IGF2R, POU3F2, and AP2A2; miR31-5p targeted CAMK2D, PPP2R2A, and YWHAE; and miR-223$3 p$ targeted PAX6.

\section{Functional enrichment analyses for miRNAs and target genes}

KEGG pathway enrichment result for miRNA was obtained. Furthermore, target genes regulated by miRNAs were significantly enriched in $14 \mathrm{KEGG}$ pathways and $40 \mathrm{GO}$-BP terms. The top 20 results are presented in Figure 3. For example, CAMK2D was enriched in amphetamine addiction, dopaminergic synapse, adrenergic signaling in cardiomyocytes, insulin secretion, circadian entrainment, CAMP signaling pathway, melanogenesis, oocyte meiosis, cholinergic synapse, and long-term potentiation. PPP2R2A was enriched in the dopaminergic synapse, adrenergic signaling in cardiomyocytes, and the Hippo signaling pathway. YWHAE was enriched in the Hippo signaling pathway and 0ocyte meiosis.

\section{Protein-protein interaction network for target genes}

The PPI network for target genes included 148 nodes and 229 relationship pairs (Fig. 4a). The module (score=5) included 5 nodes and 10 relationship pairs. The top 10 nodes with higher degrees and module nodes are presented in Table 1. The top 10 genes with higher degrees were enriched in four KEGG pathways (dopaminergic synapse, Hippo signaling pathway, adrenergic signaling in cardiomyocytes, and oocyte meiosis) and four GO-BP terms (G2/M transition of mitotic cell cycle, regulation of cellular response to heat, regulation of heart rate by cardiac conduction, and cellular response to calcium ion), and the module genes were significantly enriched in one KEGG pathway (synaptic vesicle cycle) and seven GO-BP terms (positive regulation of dendrite extension, clathrin-mediated endocytosis, calcium ion-regulated exocytosis of neurotransmitter, synaptic vesicle endocytosis, regulation of calcium ion-

Table 1. The top 10 degree nodes and module nodes in protein-protein interaction network

\begin{tabular}{|lccccc|}
\hline $\begin{array}{l}\text { Top 10 degree } \\
\text { Nodes }\end{array}$ & Description & Degree & $\begin{array}{c}\text { Module } \\
\text { Nodes }\end{array}$ & Description & Degree \\
\hline UBE2I & Up-target & 15 & SYT1 & Up-target & 10 \\
PAX6 & Down-target & 12 & AP2A2 & Down-target & 9 \\
SYT1 & Up-target & 10 & IGF2R & Down-target & 9 \\
PPP1CB & Up-target & 10 & FCHO2 & Down-target & 7 \\
AP2A2 & Down-target & 9 & SYT2 & Down-target & 6 \\
IGF2R & Down-target & 9 & & & \\
POU3F2 & Down-target & 9 & & & \\
YWHAE & Down-target & 8 & & & \\
CAMK2D & Down-target & 8 & & & \\
\hline
\end{tabular}




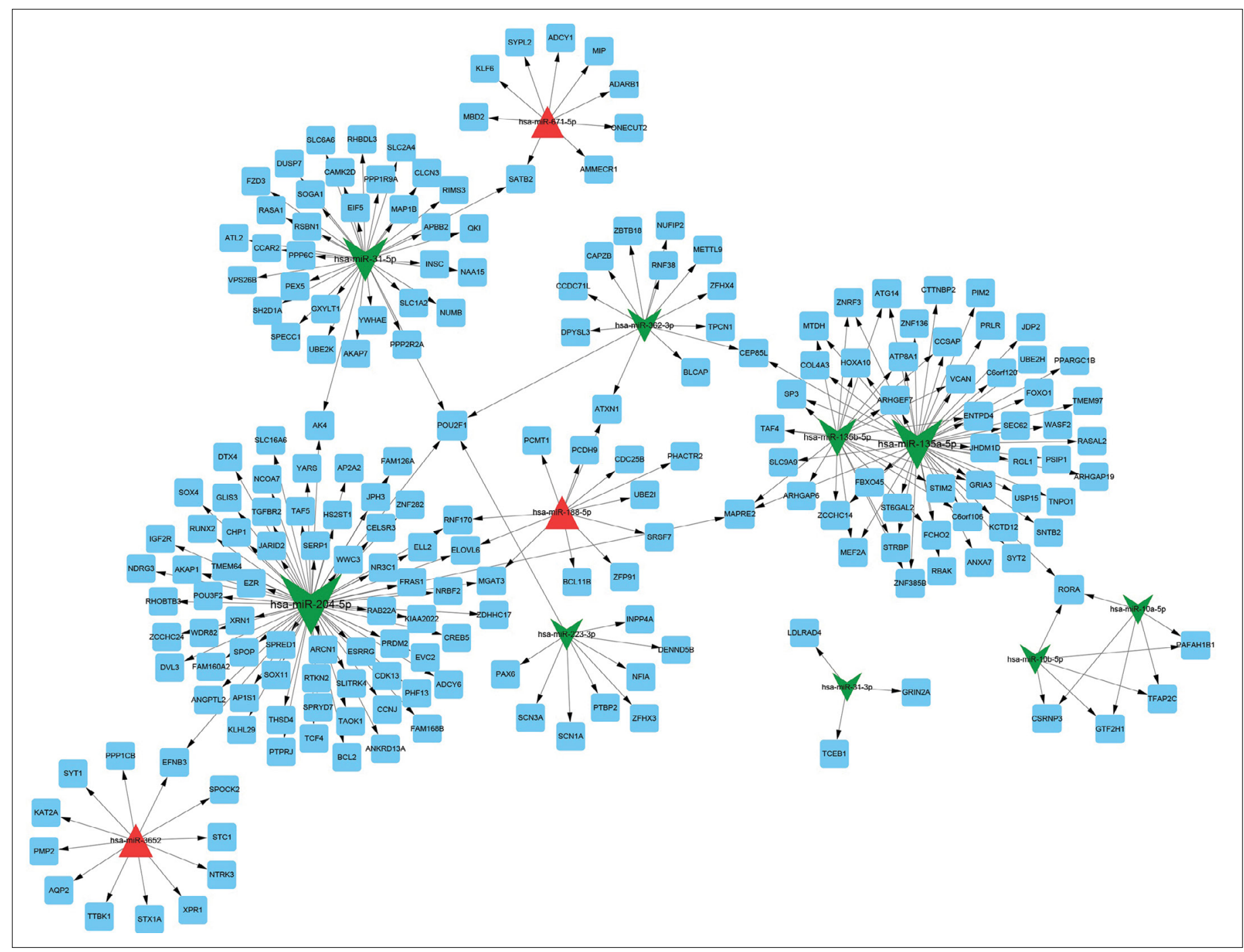

Figure 2. The miRNA-target genes regulatory network. Red triangle: upregulated miRNA; green arrow shape: downregulated miRNA; blue squares: target genes; arrowhead: regulatory direction

dependent exocytosis, neurotransmitter secretion, and vesicle fusion) (Fig. 4b).

\section{Transcription factors-target genes regulatory network}

Overall, eight TFs were predicted through WebGestalt. Totally, 22 regulatory relationship pairs were obtained through integration, of which 7 genes were regulated by downregulated miRNAs. TFs-target genes regulatory network is illustrated in Figure 5. CAMK2D was targeted by six TFs, IGF2R was targeted by four TFs, PPP2R2A was targeted by four TFs, PAX6 was targeted by three TFs, POU3F2 was targeted by two TFs, YWHAE was targeted by two TFs, and AP2A2 was targeted by one TF.

\section{Discussion}

In this study, seven genes, including CAMK2D, IGF2R, PPP2R2A, PAX6, POU3F2, YWHAE, and AP2A2, were targeted by
TFs. Among these seven genes, CAMK2D, IGF2R, PAX6, POU3F2, $Y W H A E$, and $A P 2 A 2$ belonged to the top 10 genes with higher degrees in the PPI network. Furthermore, miR-204-5p targeted IGF2R, POU3F2, and AP2A2; miR-31-5p targeted CAMK2D and YWHAE; and miR-223-3p targeted PAX6. MiR-204-5p, miR-31-5p, and miR-223-3p had more target genes. In addition, CAMK2D was significantly enriched in some pathways, such as adrenergic signaling in cardiomyocytes pathway and cAMP signaling pathway. YWHAE was signific antly enriched in the Hippo signaling pathway.

Atrial electrical and structural remodeling is the foundation of AF (26), and CaMKII had been reported to mediate the processes associated with atrial contractile function and structural remodeling in AF (27). Yao et al. (28) suggested that in pathological states, CAMK2D was associated with heart failure and myocardial hypertrophy, and CAMK2D could play a role in arrhythmia. Activation of $P A X 6$ can result in apoptosis, premature neurogenesis, and mitotic arrest (29). Jesel et al. (30) indicated 


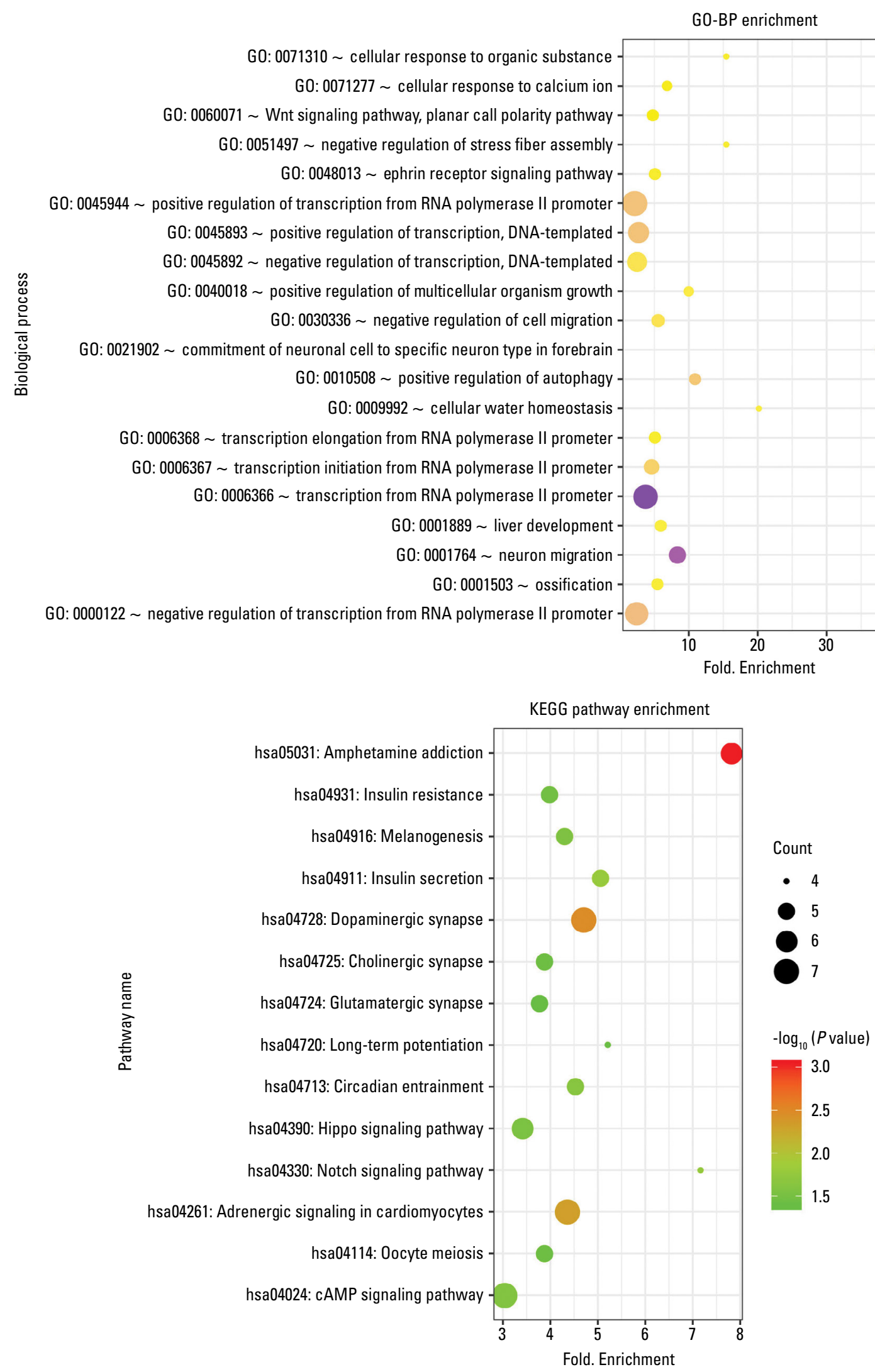

$-\log _{10}(P$ value $)$

6

5

4

3

2

Count

- 5

10

15

20

G0: 0001889 liver development

GO: $0001503 \sim$ ossification 


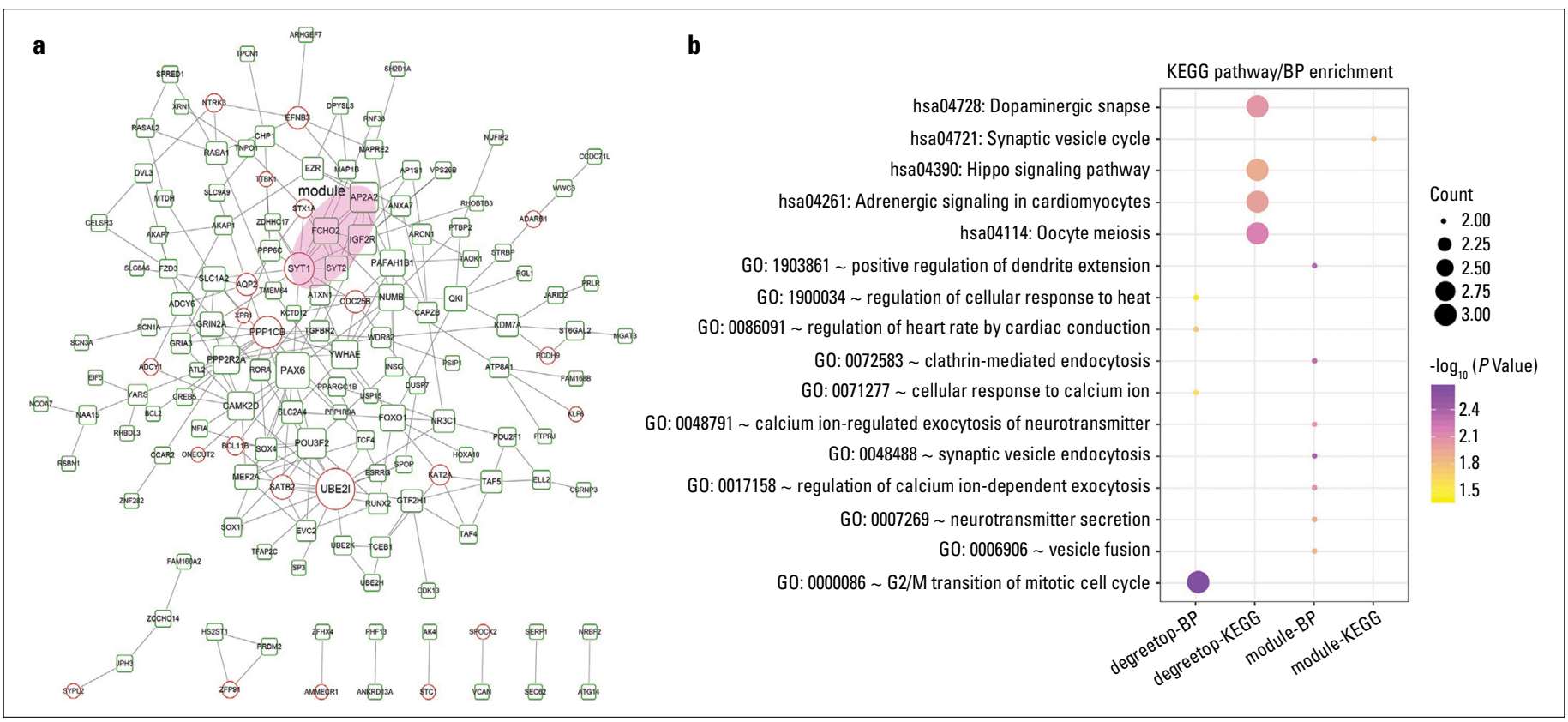

Figure 4. (a) Protein-protein interaction network for target genes; red circle: genes regulated by upregulated miRNA, green circle: genes regulated by downregulated miRNA, higher degree values indicate bigger nodes; (b) KEGG pathways and G0-BP terms for the top 10 genes with higher degrees and the module genes

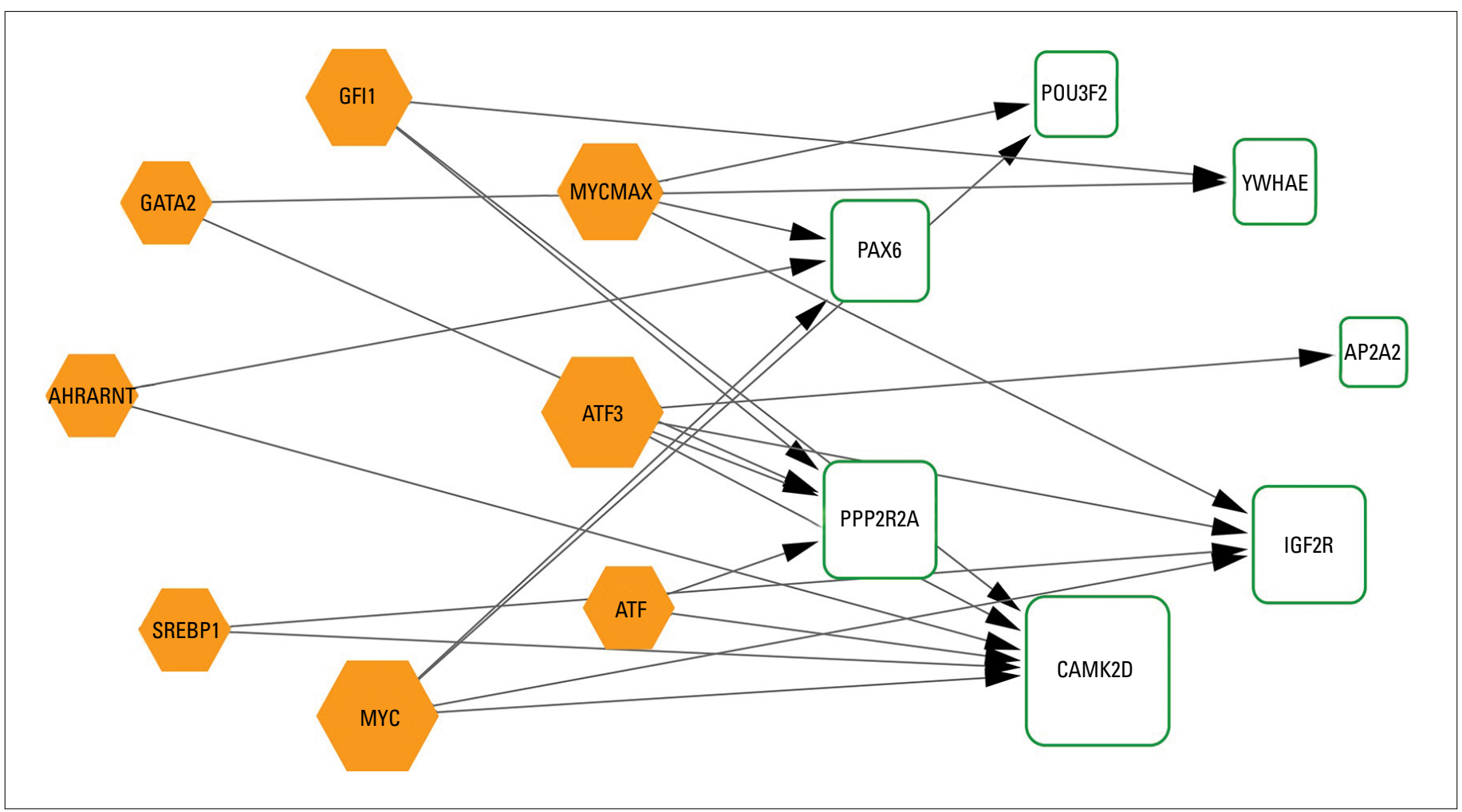

Figure 5. Transcription factors (TFs)-target genes regulatory network. Green squares: genes regulated by downregulated miRNA; Yellow hexagonal: TFs; arrowhead: regulatory direction

the significant roles of apoptosis in the progression of the prothrombotic state in AF. YWHAE (encoding 14-3-3ع) is required for ventricular morphogenesis (31). Kosaka et al. (32) reported that $14-3-3 \varepsilon$ played a significant role in the compaction of car- diac ventricles by regulating the cell cycle of the cardiomyocyte. This experiment demonstrated that both in vivo and in vitro, 14$3-3 \varepsilon$ played significant roles in cardiac channel activity $(33,34)$. $A P 2 A 2$ is a cardiac target gene of PPAR (35), suggesting the roles 
of $A P 2 A 2$ in the heart. Chu et al. (36) indicated that the inhibition of IGF2R signaling pathways might be able to prevent the development of pathological cardiac hypertrophy. Co-expression of p53 and POU4F2 may be significant for controlling pro-apoptotic gene expression in cardiomyocytes after ischemic or hypoxic insults (37). Thus, CAMK2D, IGF2R, PAX6, POU3F2, YWHAE, and $A P 2 A 2$, may be crucial in $A F$ or other diseases associated with heart, such as heart failure and arrhythmia. Taken together with our present results, we inferred that CAMK2D, IGF2R, PAX6, $P O U 3 F 2, Y W H A E$, and $A P 2 A 2$ might play essential roles in the development of $A F$.

Furthermore, in our present study, miR-204-5p targeted IGF2R, POU3F2, and AP2A2; miR-31-5p targeted CAMK2D and YWHAE; and miR-223-3p targeted PAX6. MiR-204-5p, miR-31-5p, and miR223-3p had more target genes. Reilly et al. (38) indicated that the upregulation of miR-31 might lead to atrial loss of dystrophin and nNOS in human AF. Yuan et al. (39) suggested that miR-223 might be used in the diagnosis of rheumatic heart disease, complicated with AF. Dai et al. (40) reported that the expression levels of miR-204 could be regarded as the clinical index for the diagnosis of patients with pulmonary arterial hypertension caused by congenital heart disease. Thus, we propose that miR-204-5p, miR-31-5p, and miR-223-3p may be significant miRNAs in the development of $A F$, and they play significant roles in $A F$, possibly via their target genes.

It is reported that the Hippo signaling pathway is involved in regulating the survival and proliferation of cardiomyocytes in hearts (41). IP prostanoid receptor activation inhibits cardiomyocyte hypertrophy through cAMP-dependent signaling (42), suggesting the significant role of the cAMP signaling pathway in cardiomyocyte. A previous study has reported that overexpression of cytochrome P450 family 2 subfamily $C$ member 9 (CYP2C9), a gene that plays a critical role in the variability of warfarin doses, is associated with an increase in cAMP levels, which suggested that CAMP may be related to the variability in warfarin doses. Given the therapeutic action of warfarin in $A F$, we speculated that the CAMP signaling pathway might be implicated in AF treatment through CYP2C9. In the present study, CAMK2D was significantly enriched in adrenergic signaling in cardiomyocytes pathway and cAMP signaling pathway. YWHAE was significantly enriched in the Hippo signaling pathway. Thus, we infer that $C A M K 2 D$ plays a role in $\mathrm{AF}$ via adrenergic signaling in cardiomyocytes pathway and CAMP signaling pathway affecting cardiomyocyte, and YWHAE plays a role in AF via the Hippo signaling pathway affecting cardiomyocyte.

In addition, our present study showed that CAMK2D, IGF2R, PPP2R2A, PAX6, POU3F2, YWHAE, and AP2A2 were targeted by TFs. These TFs included MYC, SREBP1, ATF, ATF3, AHRARNT, MYCMAX, GATA2, and GFI1. Among these TFs, c-Myc is a component of the VKORC1 promoter transcription factor assembly (43). Notably, VKORC1 is considered an essential biomarker for its role in contributing to high interindividual variability in warfarin anticoagulant therapy in $\mathrm{AF}$ (44). Lee et al. (7) have suggested that the allelic variant of c-Myc would elicit differences in the interaction with VKORC1, thereby resulting in an alteration of warfarin sensitivity. GATA2 belongs to the zinc-finger transcription factor family GATA that is involved in the development of blood cells, cardiac development, and cardiomyocyte differentiation $(45,46)$. It has been reported that the GATA2 transcription factor is involved in the regulation of $C Y P 2 C 9$, a vital drug-metabolizing enzyme that metabolizes warfarin (47). However, we did not find any previous studies regarding the association between the development of $A F$ and the other TFs. Hence, further studies are warranted that could explore the roles of these TFs in the development of AF.

\section{Conclusion}

In summary, miR-204-5p, miR-31-5p, and miR-223-3p could be significant miRNAs in the development of $A F$ and could prove to be essential biomarkers for its treatment. Moreover, CAMK2D, IGF2R, PAX6, POU3F2, YWHAE, and AP2A2 may be the crucial genes in the development of AF. Furthermore, miR$31-5 p \rightarrow C A M K 2 D \rightarrow$ adrenergic signaling in cardiomyocytes pathway-cAMP signaling pathway $\rightarrow$ cardiomyocytes, and miR$31-5 p \rightarrow Y W H A E \rightarrow$ Hippo signaling pathway $\rightarrow$ cardiomyocytes could be the two significant molecular mechanisms in the development of AF. Notably, miR-204 was involved in the progression of AF by regulating its target genes IGF2R, POU3F2, and $A P 2 A 2$. By contrast, miR-223-3p functioned in $A F$ by targeting PAX6, which is associated with the regulation of apoptosis in AF. However, the lack of experimental verification and a small sample size were the limitations of the current study. Therefore, further studies with large sample sizes and experimental verification are warranted to explore the molecular mechanisms of AF.

Conflict of interest: None declared.

Peer-review: Internally and externally peer-reviewed.

Funding: This work was supported by The Foundation of Key Scientific and Technological Project of Xinxiang (No. CXGG17039).

Authorship contributions: Concept - H.Z., G.Y.; Design - H.Z.; Supervision - H.Z.; Fundings - H.Z.; Materials - J.S.; Data collection and/or processing - H.Z., J.S.; Analysis and/or interpretation - X.L.; Literature search - Y.W.; Writing - H.Z.; Critical review - N.Z., Y.X., Y.Y.

\section{References}

1. Li L, Mao H, Ishwaran H, Rajeswaran J, Ehrlinger J, Blackstone $\mathrm{EH}$. Estimating the prevalence of atrial fibrillation from a threeclass mixture model for repeated diagnoses. Biom J 2017; 59: 331 43. [CrossRef] 
2. Anumonwo JM, Kalifa J. Risk factors and genetics of atrial fibrillation. Cardiol Clin 2014; 32: 485-94. [CrossRef]

3. Xiao J, Liang D, Zhao H, Liu Y, Zhang H, Lu X, et al. 2-Aminoethoxydiphenyl borate, a inositol 1,4,5-triphosphate receptor inhibitor, prevents atrial fibrillation. Exp Biol Med (Maywood) 2010; 235: 862-8. [CrossRef]

4. Oi J, Xiao J, Zhang Y, Li J, Liu Y, Li P, et al. Effects of potassium channel blockers on changes in refractoriness of atrial cardiomyocytes induced by stretch. Exp Biol Med (Maywood) 2009; 234: 779-84.

5. Nattel $S$. New ideas about atrial fibrillation 50 years on. Nature 2002; 415: 219-26. [CrossRef]

6. Duffy HS. The ever-shrinking world of cardiac ion channel remodeling: the role of microRNAs in heart disease. Heart Rhythm 2009; 6: 1810-1. [CrossRef]

7. Lee KE, Chang BC, Park S, Gwak HS. Effects of single nucleotide polymorphisms in c-Myc on stable warfarin doses in patients with cardiac valve replacements. Pharmacogenomics 2015; 16 : 1101-8. [CrossRef]

8. Feng B, Chen S, George B, Feng 0 , Chakrabarti S. miR133a regulates cardiomyocyte hypertrophy in diabetes. Diabetes Metab Res Rev 2010; 26: 40-9. [CrossRef]

9. Lu Y, Zhang Y, Wang N, Pan Z, Gao X, Zhang F, et al. MicroRNA-328 contributes to adverse electrical remodeling in atrial fibrillation. Circulation 2010; 122: 2378-87. [CrossRef]

10. Li Y, Tan W, Ye F, Xue F, Gao S, Huang W, et al. Identification of microRNAs and genes as biomarkers of atrial fibrillation using a bioinformatics approach. J Int Med Res 2019; 47: 3580-9. [CrossRef]

11. Harling L, Lambert J, Ashrafian H, Darzi A, Gooderham NJ, Athanasiou T. Elevated serum microRNA 483-5p levels may predict patients at risk of post-operative atrial fibrillation. Eur $\mathrm{J}$ Cardiothorac Surg 2017; 51: 73-8. [CrossRef]

12. Zhao $Y$, Yuan $Y$, Qiu C. Underexpression of CACNA1C Caused by Overexpression of microRNA-29a Underlies the Pathogenesis of Atrial Fibrillation. Med Sci Monit 2016; 22: 2175-81. [CrossRef]

13. Girmatsion Z, Biliczki P, Bonauer A, Wimmer-Greinecker G, Scherer M, Moritz $A$, et al. Changes in microRNA-1 expression and IK1 up-regulation in human atrial fibrillation. Heart rhythm 2009; 6: 1802-9. [CrossRef]

14. Luo X, Pan Z, Shan H, Xiao J, Sun X, Wang N, et al. MicroRNA-26 governs profibrillatory inward-rectifier potassium current changes in atrial fibrillation. J Clin Invest 2013; 123: 1939-51. [CrossRef]

15. Morishima M, Iwata E, Nakada C, Tsukamoto Y, Takanari H, Miyamoto $S$, et al. Atrial Fibrillation-Mediated Upregulation of miR-30d Regulates Myocardial Electrical Remodeling of the G-Protein-Gated K(+) Channel, IK.ACh. Circ J 2016; 80: 1346-55. [CrossRef]

16. Smyth GK. Limma: linear models for microarray data. In: Bioinformatics and Computational Biology Solutions using R and Bioconductor, R. Gentleman, V. Carey, S. Dudoit, R. Irizarry, W. Huber (eds.). Springer, New York; 2005: p.397-420. [CrossRef]

17. Wang L, Cao C, Ma $\mathrm{Q}$, Zeng $\mathrm{C}$, Wang $\mathrm{H}$, Cheng $\mathrm{Z}$, et al. RNA-seq analyses of multiple meristems of soybean: novel and alternative transcripts, evolutionary and functional implications. BMC Plant Biol 2014; 14: 169. [CrossRef]

18. Dweep H, Gretz N. miRWalk2.0: a comprehensive atlas of microRNA-target interactions. Nat Methods 2015; 12: 697. [CrossRef]

19. Shannon P, Markiel A, Ozier O, Baliga NS, Wang JT, Ramage D, et al. Cytoscape: a software environment for integrated models of biomolecular interaction networks. Genome Res 2003; 13: 2498-504.

20. Kanehisa M, Goto S. KEGG: kyoto encyclopedia of genes and genomes. Nucleic Acids Res 2000; 28: 27-30. [CrossRef]
21. Yu G, Wang LG, Han Y, He QY. clusterProfiler: an R package for comparing biological themes among gene clusters. OMICS 2012; 16: 284-7. [CrossRef]

22. Ashburner M, Ball CA, Blake JA, Botstein D, Butler H, Cherry JM, et al. Gene ontology: tool for the unification of biology. The Gene Ontology Consortium. Nat Genet 2000; 25: 25-9. [CrossRef]

23. Szklarczyk D, Franceschini A, Wyder S, Forslund K, Heller D, Huerta-Cepas J, et al. STRING v10: protein-protein interaction networks, integrated over the tree of life. Nucleic Acids Res 2015; 43: D447-52.

24. Bandettini WP, Kellman P, Mancini C, Booker OJ, Vasu S, Leung SW, et al. MultiContrast Delayed Enhancement (MCODE) improves detection of subendocardial myocardial infarction by late gadolinium enhancement cardiovascular magnetic resonance: a clinical validation study. J Cardiovasc Magn Reson 2012; 14: 83. [CrossRef]

25. Zhang B, Kirov S, Snoddy J. WebGestalt: an integrated system for exploring gene sets in various biological contexts. Nucleic Acids Res 2005; 33: W741-8. [CrossRef]

26. Igarashi T, Niwano S, Niwano H, Yoshizawa T, Nakamura H, Fukaya $\mathrm{H}$, et al. Linagliptin prevents atrial electrical and structural remodeling in a canine model of atrial fibrillation. Heart Vessels 2018; 33: 1258-65. [CrossRef]

27. Liu Z, Finet JE, Wolfram JA, Anderson ME, Ai X, Donahue JK. Calcium/calmodulin-dependent protein kinase II causes atrial structural remodeling associated with atrial fibrillation and heart failure. Heart Rhythm 2019; 16: 1080-8. [CrossRef]

28. Yao $\mathrm{Q}$, Song Z, Tong S, Wan Y, Cheng J, Ding X. e0115 Overexpression and inhibition of Camk2d gene in primary myocardial cells by lentivirus. Heart 2010; 96 (Suppl 3): A37. [CrossRef]

29. Cooper LL, Odening KE, Ziv 0, Chaves L, Schofield L, Choi BR, et al. The aging rabbit heart as a model for cardiac aging. The FASEB Journal 2010: 1 supplement: 595.6 .

30. Jesel L, Abbas M, Toti F, Cohen A, Arentz T, Morel O. Microparticles in atrial fibrillation: a link between cell activation or apoptosis, tissue remodelling and thrombogenicity. Int J Cardiol 2013; 168: 660-9.

31. Brunelli L, Cieslik KA, Michalik L, Wahli W, Yost H. Activation of PPAR delta Regulates Ywhae (Encoding 14-3-3 epsilon Protein), a Gene Required for Ventricular Morphogenesis. Basic Cardiovascular Sciences Conference 2009; 105: E15.

32. Kosaka Y, Cieslik KA, Li L, Lezin G, Maguire CT, Saijoh Y, et al. 14-3$3 \varepsilon$ plays a role in cardiac ventricular compaction by regulating the cardiomyocyte cell cycle. Mol Cell Biol 2012; 32: 5089-102.

33. Allouis M, Le Bouffant F, Wilders R, Peroz D, Schott JJ, Noireaud $\mathrm{J}$, et al. 14-3-3 is a regulator of the cardiac voltage-gated sodium channel Nav1.5. Circ Res 2006; 98: 1538-46. [CrossRef]

34. Choe CU, Schulze-Bahr E, Neu A, Xu J, Zhu Zl, Sauter K, et al. Cterminal HERG (LQT2) mutations disrupt IKr channel regulation through 14-3-3epsilon. Hum Mol Genet 2006; 15: 2888-902. [CrossRef]

35. Buroker NE, Huang JY, Barboza J, Ledee DR, Eastman RJ Jr., Reinecke $\mathrm{H}$, et al. The adaptor-related protein complex 2, alpha 2 subunit (AP2alpha2) gene is a peroxisome proliferator-activated receptor cardiac target gene. Protein J 2012; 31: 75-83. [CrossRef]

36. Chu CH, Tzang BS, Chen LM, Kuo CH, Cheng YC, Chen LY, et al. IGFII/mannose-6-phosphate receptor signaling induced cell hypertrophy and atrial natriuretic peptide/BNP expression via Galphaq interaction and protein kinase $\mathrm{C}$-alpha/CaMKII activation in $\mathrm{H} 9 \mathrm{c} 2$ cardiomyoblast cells. J Endocrinol 2008; 197: 381-90. [CrossRef]

37. Budhram-Mahadeo V, Fujita R, Bitsi S, Sicard P, Heads R. Co-expression of POU4F2/Brn-3b with p53 may be important for controlling expression of pro-apoptotic genes in cardiomyocytes following ischaemic/hypoxic insults. Cell Death Dis 2014; 5: e1503. [CrossRef] 
38. Reilly S, Liu X, Carnicer R, Rajakumar T, Sayeed R, Krasopoulos G, et al. Evaluation of the role of miR-31-dependent reduction in dystrophin and nNOS on atrial-fibrillation-induced electrical remodelling in man. Lancet 2015; 385 Suppl 1: S82. [CrossRef]

39. Yuan S, Xin XU, Fengping HE, Zhang S, Fan W, Cardiology DO. The expression and clinical value of miR-223 and MMP-9 in right atrium and blood serum in patients with atrial fibrillation. Journal of Molecular Diagnostics \& Therapy 2014; 6: 317-24.

40. Dai $H$, Yin XL. The changes of plasma miR-18a,miR-27b,miR130a,miR-204 in patients with pulmonary arterial hypertension due to congenital heart disease. J Am Coll Cardiol 2014; 64 (Supplement 21): C190. [CrossRef]

41. Robertson A, Cartwright EJ, Oceandy D. 168 Targeting The Hippo Signalling Pathway to Enhance the Therapeutic Potential of iPSDerived Cardiomyocytes. Heart 2016; 102 (Suppl 6): A118.1. [CrossRef]

42. Ritchie RH, Rosenkranz AC, Huynh LP, Stephenson T, Kaye DM, Dusting GJ. Activation of IP prostanoid receptors prevents cardio- myocyte hypertrophy via cAMP-dependent signaling. Am J Physiol Heart Circ Physiol 2004; 287: H1179-85. [CrossRef]

43. Pfister A, Osman A. The VKORC1 promoter is occupied by c-Myc transcription factor in HepG2 cells. Thromb Res 2010; 126: e150-1.

44. Wadelius M, Chen LY, Lindh JD, Eriksson N, Ghori MJ, Bumpstead $S$, et al. The largest prospective warfarin-treated cohort supports genetic forecasting. Blood 2009; 113: 784-92. [CrossRef]

45. Harigae H. GATA transcription factors and hematological diseases. Tohoku J Exp Med 2006; 210: 1-9. [CrossRef]

46. Crispino JD, Lodish MB, Thurberg BL, Litovsky SH, Collins T, Molkentin JD, et al. Proper coronary vascular development and heart morphogenesis depend on interaction of GATA-4 with FOG cofactors. Genes Dev 2001; 15: 839-44. [CrossRef]

47. Mwinyi J, Nekvindova J, Cavaco I, Hofmann Y, Pedersen RS, Landman $E$, et al. New insights into the regulation of CYP2C9 gene expression: the role of the transcription factor GATA-4. Drug Metab Dispos 2010; 38: 415-21. [CrossRef] 